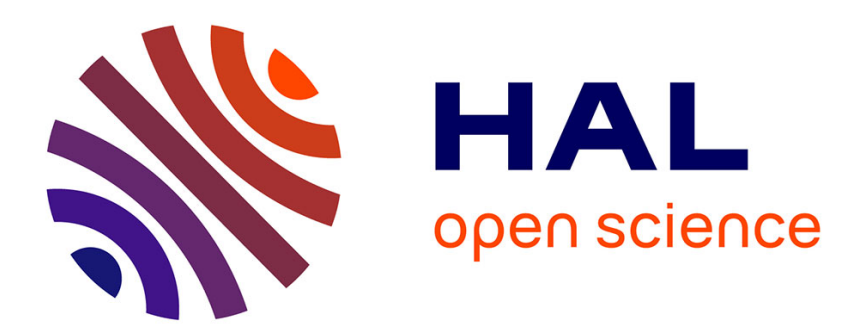

\title{
Facteurs d'échelle phénoménologiques pour la transition critique liquide-gaz des fluides purs
}

\author{
Y. Garrabos
}

\section{To cite this version:}

Y. Garrabos. Facteurs d'échelle phénoménologiques pour la transition critique liquide-gaz des fluides purs. Journal de Physique, 1985, 46 (2), pp.281-291. 10.1051/jphys:01985004602028100 jpa00209967

\section{HAL Id: jpa-00209967 https://hal.science/jpa-00209967}

Submitted on 1 Jan 1985

HAL is a multi-disciplinary open access archive for the deposit and dissemination of scientific research documents, whether they are published or not. The documents may come from teaching and research institutions in France or abroad, or from public or private research centers.
L'archive ouverte pluridisciplinaire HAL, est destinée au dépôt et à la diffusion de documents scientifiques de niveau recherche, publiés ou non, émanant des établissements d'enseignement et de recherche français ou étrangers, des laboratoires publics ou privés. 
Classification

Physics Abstracts

$64.60 \mathrm{~F}$

\title{
Facteurs d'échelle phénoménologiques pour la transition critique liquide-gaz des fluides purs
}

\author{
Y. Garrabos \\ Laboratoire des Interactions Moléculaires et des Hautes Pressions du C.N.R.S., \\ Centre Universitaire Paris-Nord, Avenue J.B. Clément, 93430 Villetaneuse, France
}

(Reçu le 3 février 1984, révisé le 2 octobre, accepté le 4 octobre 1984)

\begin{abstract}
Résumé. - Nous discutons une méthode phénologique permettant de déterminer les comportements singuliers asymptotiques d'un fluide pur à l'équilibre, lorsque son point critique liquide-vapeur et le plan tangent à la surface caractéristique en ce point sont localisés expérimentalement dans l'espace des phases pression, densité et température.
\end{abstract}

\begin{abstract}
We discuss a phenomenological method which allows to determine the singular asymptotic behaviours for a pure fluid at equilibrium, when the liquid-gas critical point and the tangent plane to the characteristic surface of this point are localized experimentally, in the pressure, density and temperature coordinates.
\end{abstract}

\section{Introduction.}

Les nombreuses applications de la théorie du groupe de renormalisation (GR) ont largement contribué à la description des phénomènes observés dans un système (S) à l'équilibre proche d'un point de transition du second ordre [1]. Des systèmes physiques très différents ayant mêmes valeurs de $d$ et de $n$, dimensionnalités respectives de l'espace et du paramètre d'ordre de la transition, ont pu ainsi être regroupés à l'intérieur d'une même classe d'universalité $[1,2]$. Pour une classe $(d, n)$ donnée, le GR prévoit seulement l'existence de deux paramètres sans dimension, caractéristiques de chaque système appartenant à cette classe $[1,3-6]$. Ce résultat vérifie l'hypothèse d'universalité à deux facteurs d'échelle près [7]. Toutefois, la théorie du GR n'est pas en mesure de déterminer les deux paramètres caractéristiques de (S).

Dans le présent travail, nous proposons une détermination phénoménologique de ces deux paramètres caractéristiques pour le cas où le système est un fluide pur à l'équilibre près de son point critique liquide vapeur $(\mathrm{S} \equiv \mathrm{PCLV})$. A cet effet, nous supposons que la description complète de ses comportements singuliers asymptotiques est entièrement définie par les quatre coordonnées suivantes : pression critique $\left(P_{\mathrm{c}}\right)$; densité critique $\left(\rho_{\mathrm{c}}\right)$; température critique $\left(T_{\mathrm{c}}\right)$; pente commune à $T_{\mathrm{c}}\left(\gamma_{\mathrm{c}}^{\prime}\right)$ de l'isochore critique et de la ligne de saturation projetées dans le diagramme $P, T$. Par conséquent les deux facteurs d'échelle caractéristiques de ce fluide pur sont reliés à ces quatre gran- deurs. Nous donnons des formes explicites de cette relation en choisissant comme facteurs d'échelle les deux amplitudes asymptotiques $\xi^{+}$et $D^{\mathrm{c}}$. $\xi^{+}$est l'amplitude de la longueur de corrélation $l^{*}$ (réduite sans dimension) définie le long de l'isochore critique dans le domaine monophasique. $D^{\mathrm{c}}$ est l'amplitude de l'isotherme critique de l'écart $\Delta \mu^{*}=\mu^{*}-\mu_{\mathrm{c}}^{*}$ de potentiel chimique par rapport à sa valeur au point critique (réduit sans dimension). L'ensemble des fluides purs constitue alors une sous-classe de la classe d'universalité $d=3, n=1[8,9]$ où la description asymptotique des phénomènes critiques est complètement déterminée. Au préalable, ceci suppose que soient utilisées pour tous les fluides purs, les mêmes définitions des grandeurs singulières réduites à des quantités sans dimension. Aussi avons-nous proposé dans ce travail, un choix explicite des deux paramètres macroscopiques dimensionnés qui sont nécessaires au passage à une description en variables sans dimension des phénomènes critiques observés au voisinage $\mathrm{du}$ (PCLV).

Dans le paragraphe suivant, nous rappelons succinctement, pour $d=3, n=1$, les résultats théoriques du GR qui sont utilisés par la suite. Le paragraphe 3 précise nos définitions des quatre grandeurs caractéristiques d'un fluide pur en termes des coordonnées $P_{\mathrm{c}}, \rho_{\mathrm{c}}, T_{\mathrm{c}}$ et $\gamma_{\mathrm{c}}^{\prime}$. Les deux premières, $\beta_{\mathrm{c}}=$ $k_{\mathrm{B}} T_{\mathrm{c}}$ et $a=\left(k_{\mathrm{B}} T_{\mathrm{c}} / P_{\mathrm{c}}\right)^{1 / 3}$, sont homogènes respectivement à une énergie et une longueur $\left(k_{\mathrm{B}}\right.$ est la constante de Boltzmann). Les deux autres, $Y_{\mathrm{c}}=\left(\gamma_{\mathrm{c}}^{\prime} T_{\mathrm{c}} / \boldsymbol{P}_{\mathrm{c}}\right)-1$ et $Z_{\mathrm{c}}=P_{\mathrm{c}} m / \rho_{\mathrm{c}} k_{\mathrm{B}} T_{\mathrm{c}}$, sont des nombres sans dimen- 
sion ( $m$ est la masse de la molécule constitutive du fluide). Nous donnons dans le paragraphe 4 les relations qui permettent le calcul des amplitudes $\xi^{+}$ et $D^{\mathrm{c}}$ d'un fluide pur dont on connaît $Y_{\mathrm{c}}$ et $Z_{\mathrm{c}}$. La comparaison avec les résultats expérimentaux existants est effectuée. Nous retrouvons ensuite ces relations en utilisant, dans le paragraphe 5, une formulation équivalente de l'universalité attendue pour la sous-classe des fluides purs. Pour le chemin d'approche du (PCLV) le long de l'isochore critique, cette formulation fait intervenir les grandeurs pression et température, directement accessibles par l'expérience. Ceci nous permet d'établir dans le dernier paragraphe une première comparaison qualitative avec les prévisions récentes de Bagnuls et Bervillier [10].

\section{Rappels des prévisions du GR.}

Les rappels théoriques qui suivent concernent les systèmes $(\mathrm{S})$ appartenant à la classe d'universalité $d=3, n=1$. Ils sont limités aux résultats caractérisant le comportement singulier asymptotique d'une propriété $X_{\mathrm{s}}$ qui est représenté par la loi de puissance non analytique suivante :

$$
X_{\mathrm{S}}(\tau)=X_{\mathrm{S}}^{(\mathrm{L})} \cdot \tau^{-x}\{1+0(\tau)\} \quad \text { avec } \tau \rightarrow 0^{(\mathrm{L})} .
$$

Toutes les quantités apparaissant dans l'équation (1) sont réduites à des grandeurs sans dimension; les prévisions propres aux termes de corrections non analytiques $0(\tau)$ prévus par Wegner [11] ne sont pas prises en considération dans ce travail [12].

La grandeur sans dimension $\tau$ est un champ pertinent $\mathrm{du}$ chemin thermodynamique suivi pour atteindre asymptotiquement le point de transition de (S); ( $\tau=0$ en ce point). Dans l'équation (1), ce chemin est symbolisé par l'index supérieur (L). Dans le cas où le système $(\mathrm{S})$ est un fluide pur, $\tau$ pourrait être, par exemple, l'écart réduit de température $\left(T-T_{\mathrm{c}}\right) / T_{\mathrm{c}} ;(\mathrm{L})$ serait alors l'isochore critique, représentée habituellement par les index supérieurs + et - suivant que sont considérés le domaine monophasique $\left(T>T_{\mathrm{c}} ; \tau>0\right)$ et le domaine à deux phases, liquide et vapeur $\left(T<T_{\mathrm{c}} ; \tau<0\right)$. L'exposant critique $x$ est un nombre universel, c'est-à-dire ne dépendant pas de $(\mathrm{S})$ mais seulement du couple $(d, n)$. L'amplitude asymptotique correspondante, $X_{\mathrm{S}}^{(\mathrm{L})}$, est un nombre sans dimension qui dépend du système $(S)$ étudié.

Pour $d=3, n=1$, les résultats numériques du GR, obtenus par le traitement du modèle $\phi^{4}$ à trois dimensions ( $\left.\mathrm{S} \equiv \phi^{4} 3 \mathrm{D}\right)[3,5]$, sont très précis et vérifient l'hypothèse d'universalité à deux facteurs d'échelle près. Pour $\left(\phi^{4} 3 \mathrm{D}\right)$, ceci se traduit par l'existence de seulement dix rapports universels entre les douze amplitudes asymptotiques comme il existe dix lois d'échelle reliant les douze exposants cri- tiques de cette classe d'universalité [13]. En conséquence, la non-universalité d'un système (S) appartenant à $d=3, n=1$ se caractérise par un choix adéquat de deux amplitudes asymptotiques indépendantes. Dans le présent travail, nous avons choisi le couple formé par les deux amplitudes $\xi^{+}$et $D^{\text {c }}$ définies par les lois suivantes :

i) le long de l'isochore critique monophasique $\left(\tau>0 ; h_{\mathrm{S}}=0 ;(\mathrm{L}) \equiv+\right)$ :

$$
l_{\mathrm{S}}=\xi^{+} \cdot \tau^{-v}
$$

ii) le long de l'isotherme critique $\left(\tau=0 ; h_{\mathrm{S}} \neq 0\right.$; $(\mathrm{L}) \equiv \mathrm{c})$ :

$$
h_{\mathrm{S}}=D^{\mathrm{c}} \cdot m_{\mathrm{S}}^{\delta}
$$

où $v$ et $\delta$ sont les deux exposants critiques correspondants ; $m_{\mathrm{S}}$ est le paramètre d'ordre de la transition; $h_{\mathrm{S}}$ est le champ conjugué du paramètre d'ordre; $l_{\mathrm{s}}$ est la longueur de corrélation des fluctuations du paramètre d'ordre. Dans le cas où le système (S) est un fluide pur, les grandeurs dimensionnées correspondantes sont, d'une part, l'écart de densité en nombre à la densité en nombre critique, $\left(\rho-\rho_{\mathrm{c}}\right) / m$, comme paramètre d'ordre et, d'autre part, l'écart de potentiel chimique par particule par rapport à sa valeur au point critique, $\left(\mu-\mu_{\mathrm{c}}\right) \cdot m$, comme champ conjugué [14].

Dans le tableau I, nous avons adopté une présentation des résultats du GR [15] où on peut vérifier aisément que la connaissance des deux nombres sans dimension $\xi^{+}$et $D^{c}$ d'un système $(\mathrm{S})$ suffit bien à caractériser complètement tous ses autres comportements critiques asymptotiques. Les valeurs théoriques respectives des deux exposants indépendants $v$ et $\delta$ de la classe considérée sont $v=0,63, \delta=4,815$ [4].

Soulignons toutefois que cette présentation de l'universalité prédite pour une classe donnée n'est que la conséquence de l'hypothèse de base de l'approche du GR [16]. Cette approche suppose en effet des relations analytiques entre les deux champs pertinents renormalisés $t^{*}$ et $h^{*}$ du modèle $\left(\phi^{4} 3 \mathrm{D}\right)$ et les champs physiques correspondants $\tau$ et $h_{\mathrm{s}}$ du système ( $S$ ) qui va être représenté par ce modèle. Asymptotiquement au point de transition ces relations s'écrivent :

$$
\begin{aligned}
& t^{*}=\theta \tau+0\left(\tau^{2}, \tau h_{\mathrm{S}}\right) \\
& h^{*}=\psi h_{\mathrm{S}}+0\left(h_{\mathrm{S}}^{2}, \tau h_{\mathrm{S}}\right)
\end{aligned}
$$

où $\theta$ et $\psi$ sont nécessairement non universels. Le concept d'universalité à deux facteurs d'échelle près reflète directement la linéarisation des équations (4) et (5), selon laquelle chaque système (S) d'une même classe d'universalité est caractérisé uniquement par deux échelles, $\theta$ et $\psi$, reliées respectivement à $\tau$ et à $h_{\mathrm{S}}[16,10]$. Il est donc équivalent de caractériser la non-universalité d'un fluide, soit par les deux amplitudes $\xi^{+}$et $D^{\mathrm{c}}$, soit par les deux facteurs d'échelle $\theta$ et $\psi[17]$. 


\section{Paramètres caractéristiques du (PCLV).}

Les propriétés thermodynamiques singulières d'un fluide pur sont habituellement réduites sans dimension en utilisant $P_{\mathrm{c}}, \rho_{\mathrm{c}}$ et $T_{\mathrm{c}}$ [18]. Ce procédé présente toutefois des inconvénients [19]. Ainsi, le résultat obtenu est-il toujours défini au facteur de compressibilité près, alors que ce nombre sans dimension, $Z_{\mathrm{c}}$, défini par le rapport

$$
Z_{\mathrm{c}}=\frac{P_{\mathrm{c}} m}{\rho_{\mathrm{c}} k_{\mathrm{B}} T_{\mathrm{c}}},
$$

est caractéristique de chaque fluide pur. De plus, la connaissance des trois paramètres critiques $P_{\mathrm{c}}, \rho_{\mathrm{c}}$, $T_{\mathrm{c}}$ n'est pas suffisante pour définir thermodynamiquement les lignes d'approche asymptotique de ce point de transition. En effet, la définition univoque de ces lignes nécessite non seulement de localiser le point critique dans l'espace des phases, mais aussi de positionner le plan tangent en ce point à la surface caractéristique. Précisons cela en considérant la surface caractéristique habituelle aux expérimentateurs, définie par l'équation d'état $f(P, \rho, T)=0$.

Le plan tangent au point critique à cette surface caractéristique est défini par deux directions orthogonales passant par ce point. L'une de ces directions est triviale et commune à tous les fluides purs puisqu'il s'agit de la direction de l'axe des densités; la tangente à l'isotherme critique au point critique lui est parallèle. A l'inverse, la deuxième direction est caractéristique de chaque fluide pur. Elle est définie comme pente commune au point critique à l'isochore critique $\rho=\rho_{\mathrm{c}}$ et à la courbe de pression de vapeur saturante $P_{\mathrm{S}}(T)$, projetées sur le diagramme $(P, T)$. Nous l'écrivons :

$$
\gamma_{\mathrm{c}}^{\prime}=\frac{\partial}{\partial T}\left[P\left(T, \rho_{\mathrm{c}}\right)\right]_{T \rightarrow T_{\mathrm{c}}^{+}}=\frac{\mathrm{d}}{\mathrm{d} T}\left[P_{\mathrm{S}}(T)\right]_{T \rightarrow T_{\overline{\mathrm{c}}}^{-}} .
$$

$\mathrm{Du}$ point de vue de l'approche thermodynamique du (PCLV), les coordonnées macroscopiques sont donc au nombre de quatre. A partir de ces quatre paramètres critiques dimensionnés, nous définissons quatre nouvelles grandeurs équivalentes par les relations suivantes [19] :

$$
\begin{aligned}
& \beta_{\mathrm{c}}=k_{\mathrm{B}} T_{\mathrm{c}} \\
& a=\left(k_{\mathrm{B}} T_{\mathrm{c}} / P_{\mathrm{c}}\right)^{1 / 3} \\
& Z_{\mathrm{c}}=P_{\mathrm{c}} m / \rho_{\mathrm{c}} k_{\mathrm{B}} T_{\mathrm{c}} \\
& Y_{\mathrm{c}}=\left(\gamma_{\mathrm{c}}^{\prime} T_{\mathrm{c}} / P_{\mathrm{c}}\right)-1
\end{aligned}
$$

où $\beta_{\mathrm{c}}$ et $a$ sont les deux seules quantités dimensionnées respectivement homogènes à une énergie et à une longueur définies au point critique. Elles sont suffisantes pour réduire sans dimension toutes les grandeurs physiques du fluide pur correspondant [20]. $Z_{\mathrm{c}}$ et $Y_{\mathrm{c}}$ sont deux nombres sans dimension caractéristiques de chaque fluide.

Les analogies thermodynamiques entre systèmes appartenant à une même classe d'universalité $[8,9]$ sont à la base du choix des relations (8b) et (8d). Ces analogies s'établissent à partir des propriétés de symétrie macroscopique des surfaces caractéristiques [9]. Le long de l'isochore critique dans le domaine monophasique, le champ $P / T$ dans le cas des fluides purs est la variable analogue de la densité d'énergie libre de Gibbs dans le cas des systèmes d'Ising [21, 22]. La réduction habituelle de ce champ par le rapport correspondant des paramètres critiques conduit à la définition (8b) de $a$ comme unité de longueur. Quant à la forme donnée à $Y_{\mathrm{c}}$ (Eq. (8d)), elle tient compte du fait que le comportement asymptotique divergent de la chaleur spécifique le long de ce chemin dérive justement de la contribution singulière à ce champ $P / T[21,23]$.

Soulignons que les nombres sans dimension $Z_{\mathrm{c}}$ et $Y_{\mathrm{c}}$ ne sont pas deux des douze amplitudes critiques asymptotiques de la classe d'universalité $d=3, n=1$, définies dans le tableau I.

Comme tous les fluides purs doivent présenter l'universalité prévue par le (GR) conformément à l'hypothèse faite dans notre introduction, nous admettons qu'il existe entre $Z_{\mathrm{c}}$ et $Y_{\mathrm{c}}$ d'une part, et $\xi^{+}$et $D^{\mathbf{c}}$ d'autre part, les dépendances fonctionnelles suivantes:

$$
\begin{aligned}
\xi^{+} & =\xi^{+}\left(Z_{c}, Y_{c}\right) \\
D^{\mathrm{c}} & =D^{\mathrm{c}}\left(Z_{\mathrm{c}}, Y_{\mathrm{c}}\right) .
\end{aligned}
$$

Il ressort de cette nouvelle hypothèse une caractéristique topologique importante. Les relations (9) supposent que toute l'information nécessaire à la description asymptotique des comportements divergents à l'approche du (PCLV) est contenue dans la localisation dans l'espace des phases, de ce point sur la surface caractéristique et du plan tangent en ce point à cette surface.

\section{Détermination phénoménologique de $\xi^{+}$et $D^{c}$.}

Le choix des amplitudes $\xi^{+}$et $D^{\mathrm{c}}(\S 2)$ privilégie deux chemins d'approche du (PCLV) qui sont thermodynamiquement distincts puisque correspondant l'un à l'isochore critique, l'autre à l'isotherme critique.

Une simplification notable des équations (9) est envisageable si $Z_{\mathrm{c}}$ et $Y_{\mathrm{c}}$ interviennent séparément sur chacun de ces deux chemins. A l'évidence $Y_{c}$ doit être lié à l'isochore critique puisque c'est la valeur réduite au point critique de la pente du potentiel $\left(P / k_{\mathrm{B}} T\right)$ défini le long de ce chemin. Pour établir un lien entre $Z_{\mathrm{c}}$ et l'isotherme critique, remarquons en préalable que, d'après le GR, l'amplitude $D^{\mathfrak{c}}$ est liée de manière univoque à l'amplitude $\hat{D}$ par le rapport universel $R_{\mathrm{D}}$ (Tableau I). Or cette dernière amplitude correspond à une grandeur divergente au point critique lui-même $\left(t^{*}=0 ; h^{*}=0\right)$. Par conséquent, $Z_{\mathrm{c}}$ et $\hat{D}$ sont les seuls nombres définis uniquement au point critique. Dès lors, il reste légitime d'associer le rôle spécifique de $Z_{\mathrm{c}}$ à l'isotherme critique dans la mesure où la direction du plan tangent 
Tableau I. - Equivalence des rapports universels et des lois d'échelle reliant respectivement les amplitudes critiques asymptotiques et les exposants critiques correspondants de la classe d'universalité $d=3, n=1$. Les définitions et notations sont celles de la référence [13], sauf pour les quatre nouveaux rapports universels $Q_{\mathrm{A}^{\mathrm{c}}}, Q_{\mathrm{C}^{+}}, Q_{\mathrm{B}}$ et $Q_{\xi^{\mathrm{c}}}$ introduits dans la partie supérieure de ce tableau. Ils sont évidemment reliés aux autres rapports universels proposés dans la référence [13] comme le montre la partie b du tableau I.

[Analogy between the universal ratios and the scaling laws respectively connecting the asymptotic amplitudes and the critical exponents, for the $d=3, n=1$ universality class. Definitions and notations are those used in reference [13], except for the four new universal ratios $Q_{\mathrm{A}^{\mathrm{c}}}, Q_{\mathrm{C}^{+}}, Q_{\mathrm{B}}$ and $Q_{\xi^{\mathrm{c}}}$, which have been introduced in the upper part of this table. Of course, they are connected to the other universal ratios proposed in reference [13], as shown in part b of table I.]

\begin{tabular}{|c|c|c|c|c|c|}
\hline \multicolumn{2}{|c|}{$\begin{array}{l}\text { a. Classe d'universalité } \\
\qquad d=3, \quad n=1\end{array}$} & $\begin{array}{l}\text { Ampli- } \\
\text { tudes }\end{array}$ & Rapports universels & $\begin{array}{l}\text { Expo- } \\
\text { sants }\end{array}$ & Lois d'Echelle \\
\hline \multirow{2}{*}{ 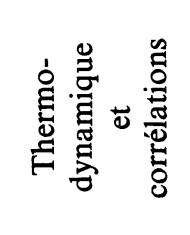 } & $\begin{array}{l}\text { Grandeurs } \\
\text { indépendantes }\end{array}$ & $\begin{array}{l}\xi^{+} \\
D^{\mathrm{c}}\end{array}$ & & $\begin{array}{l}v=0,63 \\
\delta=4,815\end{array}$ & \\
\hline & Universalité & $\begin{array}{l}A^{+} \\
\hat{D}\end{array}$ & $\begin{aligned} R_{\xi}^{+} & =\xi^{+} \cdot\left(A^{+}\right)^{1 / d} \\
R_{\mathrm{D}} & =D^{\mathrm{c}} \cdot(\hat{D})^{(\delta+1) / 2}\end{aligned}$ & $\begin{array}{l}\alpha \\
\eta\end{array}$ & $\begin{aligned} d v & =2-\alpha \\
(2-\eta) / d & =(\delta-1) /(\delta+1)\end{aligned}$ \\
\hline \multirow[b]{2}{*}{ 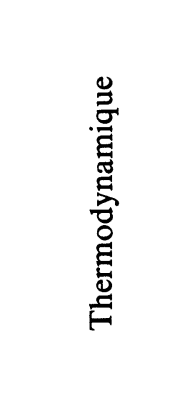 } & $\begin{array}{c}\text { Grandeurs } \\
\text { indépendantes }\end{array}$ & $D^{\mathrm{c}}, A^{+}$ & & $\delta, \alpha$ & \\
\hline & Universalité & $\begin{array}{l}C^{\mathrm{c}} \\
A^{\mathrm{c}} \\
C^{+} \\
B \\
C^{-} \\
A^{-}\end{array}$ & $\begin{array}{c}\mathrm{i}=\delta \cdot C^{\mathrm{c}} \cdot\left(D^{\mathrm{c}}\right)^{1 / \delta} \\
Q_{\mathrm{A}^{\mathrm{c}}}=A^{\mathrm{c}} \cdot\left[\delta \cdot A^{+} \cdot\left(D^{\mathrm{c}}\right)^{1 / \delta}\right]^{-\alpha /(2-\alpha)} \\
Q_{\mathrm{C}^{+}}=C^{+} \cdot\left[\left(A^{+}\right)^{\delta-1} \cdot\left(D^{\mathrm{c}}\right)^{2}\right]^{1 / \delta} \\
Q_{\mathrm{B}}=B \cdot\left(D^{\mathrm{c}} / A^{+}\right)^{1 /(\delta+1)} \\
C^{+} / C^{-} \\
A^{+} / A^{-}\end{array}$ & $\begin{array}{l}\gamma^{\mathbf{c}} \\
\alpha^{\mathbf{c}} \\
\gamma \\
\beta \\
\gamma^{\prime} \\
\alpha^{\prime}\end{array}$ & $\begin{aligned} \gamma^{\mathfrak{c}} & =(\delta-1) / \delta \\
\alpha^{\mathrm{c}} & =\alpha(\delta+1) /(2-\alpha) \\
\gamma & =(2-\alpha) \cdot(\delta-1) /(\delta+1) \\
\beta & =(2-\alpha) /(\delta+1) \\
\gamma & =\gamma^{\prime} \\
\alpha & =\alpha^{\prime}\end{aligned}$ \\
\hline \multirow{2}{*}{ 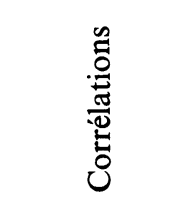 } & $\begin{array}{c}\text { Grandeurs } \\
\text { indépendantes }\end{array}$ & $\xi^{+}, \hat{D}$ & & $v, \eta$ & \\
\hline & Universalité & $\begin{array}{l}\xi^{\mathrm{c}} \\
\xi^{-}\end{array}$ & $\begin{array}{c}Q_{\xi^{\mathrm{c}}}=\xi^{\mathrm{c}} \cdot(\hat{D})^{(\delta+1) / 2 d \delta} \\
\xi^{+} / \xi^{-}\end{array}$ & $\begin{array}{l}v^{\mathrm{c}} \\
v^{\prime}\end{array}$ & $\begin{aligned} v^{\mathrm{c}} & =2 /(d+2-\eta) \\
v & =v^{\prime}\end{aligned}$ \\
\hline \multicolumn{6}{|c|}{ b. Relations avec les rapports universels du tableau 1 de la référence [13] : } \\
\hline \multicolumn{6}{|c|}{$\begin{aligned} Q_{\mathrm{A}^{\mathrm{c}}} & =\left[R_{\mathrm{A}^{\mathrm{c}}} /\left(R_{\xi}^{+}\right)^{2 d}\right]^{-\alpha /(2-\alpha)} \\
Q_{\mathrm{C}^{+}} & =\left[\left(R_{\chi}\right)^{2} \cdot\left(R_{\mathrm{C}}\right)^{\delta-1}\right]^{1 / \delta} \\
Q_{\mathrm{B}} & =\left(R_{\chi} / R_{\mathrm{C}}\right)^{1 /(\delta+1)} \\
Q_{\xi^{\mathrm{c}}} & =\left[Q_{2} \cdot Q_{3} / \delta \cdot\left(R_{\mathrm{D}}\right)^{1 / \delta}\right]^{2 \delta /(\delta-1)}\end{aligned}$} \\
\hline
\end{tabular}

le long de cette ligne est la même pour tous les fluides purs. Nous étendons donc l'importance de ces observations phénoménologiques en admettant les deux hypothèses supplémentaires suivantes :

i) Le nombre sans dimension $Y_{\mathrm{c}}$ est caractéristique d'au moins un comportement singulier asymptotique le long de l'isochore critique.

ii) Le nombre sans dimension $Z_{\mathrm{c}}$ est caractéristique d'au moins un comportement singulier asymptotique le long de l'isotherme critique.

D'où les modifications suivantes des équations (9) :

$$
\begin{aligned}
& \xi^{+}=\xi^{+}\left(Y_{\mathrm{c}}\right) \\
& D^{\mathrm{c}}=D^{\mathrm{c}}\left(Z_{\mathrm{c}}\right) .
\end{aligned}
$$

Les arguments phénoménologiques et théoriques qui permettent d'expliciter les fonctionnelles ci-dessus seront avancés dans les paragraphes suivants. Mais pour souligner dès à présent leur intérêt pratique vis-à-vis de la sous-classe des fluides purs, nous écrivons ces formes, sans autre justification que celle liée à leur simplicité, de la façon suivante :

$$
\begin{aligned}
& \left(\xi^{+}\right)^{\phi} \cdot Y_{\mathrm{c}}=Y_{\mathrm{G}} \\
& \left(D^{\mathrm{c}}\right)^{\zeta} \cdot Z_{\mathrm{c}}=F_{\mathrm{G}}
\end{aligned}
$$

où $\phi, \zeta, Y_{\mathrm{G}}$ et $F_{\mathrm{G}}$ ont les mêmes valeurs respectives pour tous les fluides purs. Sachant que les valeurs expérimentales de $Z_{\mathrm{c}}$ et $Y_{\mathrm{c}}$ sont accessibles pour de 
nombreux fluides, les équations (11) contiennent encore six grandeurs inconnues $\left(\xi^{+}, D^{\mathrm{c}}, \phi, \zeta, Y_{\mathrm{G}}, F_{\mathrm{G}}\right)$.

En principe, il suffit de connaître les valeurs expérimentales de $\xi^{+}$et de $D^{\text {c }}$ pour seulement deux de ces fluides ayant des nombres $Y_{\mathrm{c}}$ et $Z_{\mathrm{c}}$ différents pour que les grandeurs $\phi, \zeta, Y_{\mathrm{G}}$ et $F_{\mathrm{G}}$ soient aussi déterminées. A ce propos, soulignons que le caractère asymptotique des amplitudes $\xi^{+}$et $D^{\mathbf{c}}$ impose des expériences effectuées dans la région la plus proche possible du (PCLV); ainsi, les valeurs obtenues sont très faiblement affectées par les contributions des termes correctifs $[14,24]$ aux lois de divergence pure qui définissent ces amplitudes.

Les conditions précédentes sont réalisées pour les trois fluides $\mathrm{Xe}, \mathrm{SF}_{6}$ et $\mathrm{CO}_{2}$, avec les résultats des expériences de diffraction de Franhauffer [25] d'une part et de diffusion de la lumière [26-29] d'autre part. Toutefois, la valeur de l'amplitude $D^{\mathrm{c}}$ qui est déduite des expériences de diffraction de Franhauffer reste entachée d'une erreur importante reflétant l'impossibilité d'observer directement la forme de l'isotherme critique du potentiel chimique d'un fluide pur [30].

C'est pourquoi il est préférable d'ajouter une étape complémentaire permettant le calcul de $D^{\text {c }}$ sans que les conditions précédentes d'accès aux grandeurs $\phi, \zeta, Y_{\mathbf{G}}$ et $F_{\mathbf{G}}$ soient modifiées dans leur principe. En effet, en plus de la valeur de $\xi^{+}$, celles des amplitudes $C^{+}$et $B$ sont aussi obtenues avec une précision acceptable (de l'ordre de quelques pour cent) à partir des deux types d'expériences considérées. $\mathrm{C}^{+}$est l'amplitude de la divergence de la susceptibilité isotherme le long de l'isochore critique dans le domaine monophasique. $B$ est l'amplitude de la forme du sommet de la courbe de coexistence liquidevapeur du domaine à deux phases. Nous pouvons alors utiliser la valeur théorique du rapport universel $R_{\chi}$ (Tableau I) pour obtenir $D^{\text {c }}$ connaissant $B$ et $C^{+}$. Plus généralement, en choisissant parmi les valeurs expérimentales des amplitudes $\xi^{+}, B$ et $C^{+}$, un couple quelconque comme données indépendantes, l'amplitude restante et l'amplitude $D^{\text {c }}$ sont toujours calculables à partir des deux rapports universels suivants (Tableau I) :

$$
\begin{gathered}
R=R_{\xi}^{+} \cdot\left(R_{\mathrm{C}}\right)^{-1 / d}=\xi^{+} \cdot\left(B^{2} / C^{+}\right)^{1 / d} \\
R_{\chi}=D^{\mathrm{c}} \cdot B^{\delta-1} \cdot C^{+}
\end{gathered}
$$

en utilisant les valeurs théoriques $R_{\xi}^{+}=0,27$, $R_{\mathrm{C}}=0,066, R_{\chi}=1,7[5]$.

En pratique, nous avons choisi le Xe comme fluide de référence de la sous-classe des fluides purs. Les rôles spécifiques de $Y_{\mathrm{c}}$ et de $Z_{\mathrm{c}}$ sont dégagés de la comparaison $\mathrm{Xe}_{\mathrm{SF}}\left(Y_{\mathrm{c}}\right.$ intervenant seul$)$, et de celle $\mathrm{Xe}-\mathrm{CO}_{2}$ ( $Y_{\mathrm{c}}$ et $Z_{\mathrm{c}}$ intervenant simultanément). Dans ces conditions, en utilisant tous les couples possibles formés avec les valeurs expérimentales des trois amplitudes asymptotiques $B, C^{+}$et $\xi^{+}$, nous obtenons tout d'abord les valeurs empiriques suivantes des exposants $\phi$ et $\zeta$ :

$$
\begin{aligned}
& \phi=1,55 \pm 0,15 \\
& \zeta=-0,12 \pm 0,06
\end{aligned}
$$

L'incertitude sur l'exposant $\zeta$, liée à sa petitesse, reflète deux conditions défavorables pour sa détermination; i) les faibles différences relatives entre les valeurs de $Z_{\mathrm{c}}$ des trois fluides examinés ; ii) la variation relative importante de la valeur centrale de $D^{c}$ calculée pour chacun d'entre eux en fonction des valeurs expérimentales des deux amplitudes qui ont été choisies comme base indépendante.

A ce jour, il existe un trop petit nombre d'expériences sur des fluides différents très près de leur (PCLV) pour que, les équations (11) étant admises empiriquement, nous puissions encore accroître la précision de la détermination des exposants $\phi$ et $\zeta$ par la méthode précédente. Mais notre démarche phénoménologique implique d'autres contraintes sur $\phi$ et $\zeta$ qui permettent de mieux définir leurs valeurs centrales empiriques (Eqs. (14)). Ceci parce que l'universalité de la sous-classe des fluides purs est nécessairement celle prédite par le $(\mathrm{GR})$, pour la classe complète $(3,1)$. Par conséquent, les deux seuls exposants critiques indépendants sont $v$ et $\delta$, définis au paragraphe 2 (Tableau I). Ce qui, compte tenu des deux hypothèses formulées au début de ce paragraphe, suppose des relations entre, d'une part $\phi$ et $v$ le long de l'isochore critique, et, d'autre part $\zeta$ et $\delta$ le long de l'isotherme critique. Ici encore, nous donnons immédiatement les formes explicites de ces relations :

$$
\begin{array}{ll}
\phi=1 / v, & (v=0,63 ; \phi=1,587) \\
\zeta=-2 /[3(\delta+1)], & (\delta=4,815 ; \zeta=-0,11465)
\end{array}
$$

en attendant que les considérations ci-dessus soient approfondies dans les paragraphes 5 et 6 lorsque nous discuterons les transformations d'échelle des mesures de la distance au (PCLV) sur chacun de ces chemins. Soulignons déjà, que le récent résultat de Bagnuls et Bervillier [10] décrivant le comportement de la longueur de corrélation le long de l'isochore critique rend évidente la relation $\phi=1 / v$ (Eq. (15a)).

Avec les valeurs de $\phi$ et $\zeta$ données par les équations (15), nous obtenons ensuite les constantes $Y_{\mathrm{G}}$ et $F_{\mathrm{G}}$ en utilisant les valeurs des amplitudes $B$ et $C^{+}$du fluide de référence (xénon) [34] :

$$
\begin{aligned}
& Y_{\mathrm{G}}=0,380 \\
& F_{\mathrm{G}}=0,526 .
\end{aligned}
$$

Une première justification partielle des équations phénoménologiques (11), (15) et (16) est fournie par les tableaux II et III.

Dans le tableau II nous comparons, pour une vingtaine de fluides purs, les valeurs de l'amplitude dimensionnée $\xi_{0}^{+}=\xi^{+} . a$ obtenues à partir des 
Tableau II. - Valeurs (exprimées en $\AA$ ) de l'amplitude critique asymptotique $\xi_{0}^{+}$de la longueur de corrélation le long de l'isochore critique $\left(T>T_{\mathrm{c}}\right)$ pour différents fluides purs.

$1^{\mathrm{re}}$ colonne : notre détermination de $\xi_{0}^{+}=a . \xi^{+} \grave{a}$ partir des équations (8b), (8d), (11a), (15a), (16a) proposées dans ce travail.

$2^{\mathrm{e}}$ colonne : valeurs calculées semi-empiriquement par Sengers et al. (Réf. [35]).

3e colonne : valeurs expérimentales tirées des mesures d'intensité de lumière diffusée à $90^{\circ}$ et des mesures de turbidité effectuées au L.I.M.H.P. par Y. Garrabos, $R$. Tufeu et B. Le Neindre (Réf. [29]).

$4 \mathrm{e}$ colonne : valeurs expérimentales utilisées par Sengers et Moldover dans la référence [32] et par Sengers dans la référence [33] (pour les expériences qui sont à l'origine de ces valeurs, voir les Réfs. [26] à [29]).

[Values in $\AA$ of the asymptotic critical amplitude $\xi_{0}^{+}$ of the correlation length along the critical isochore $\left(T>T_{\mathrm{c}}\right.$ ) for a number of pure fluids.

Column 1 : our determination using equations $(8 b)$, (8d), (11a), (15a) and (16a) with $\xi_{0}^{+}=a . \xi^{+}$.

Column 2 : calculated values in a semi-empirical way by Sengers et al. (see Ref. [35]).

Column 3 : experimental values obtained by light scattering intensity measurements and turbudity measurements by Garrabos Y., Tufeu R. and Le Neindre B. in the L.I.M.H.P. (see Ref. [29]).

Column 4 : experimental values reported by Sengers and Moldover in reference [32] and by Sengers in reference [33] (for original references of the experimental works see Refs. [26-29]).]

\begin{tabular}{|c|c|c|c|c|}
\hline & \multicolumn{4}{|c|}{$\xi_{0}^{+}\left(10^{-10} \mathrm{~m}\right)$} \\
\hline & 1 & 2 & 3 & 4 \\
\hline $\mathrm{Ar}$ & 1,50 & 1,61 & & \\
\hline $\mathrm{Kr}$ & 1,61 & 1,73 & & \\
\hline $\mathrm{Xe}$ & 1,76 & 1,89 & $1,85 \pm 0,1$ & $1,86 \pm 0,1$ \\
\hline $\mathrm{CO}_{2}$ & 1,46 & 1,56 & $1,5 \pm 0,1$ & $1,50 \pm 0,1$ \\
\hline $\mathrm{SF}_{6}$ & 1,84 & 1,96 & $1,9 \pm 0,1$ & $1,86 \pm 0,1$ \\
\hline $\mathrm{C}_{2} \mathrm{H}_{6}$ & 1,80 & 1,84 & $1,8 \pm 0,1$ & \\
\hline $\mathrm{CClF}_{6}$ & 1,82 & & $1,95 \pm 0,15$ & \\
\hline $\mathrm{NH}_{3}$ & 1,35 & 1,44 & & \\
\hline $\mathrm{C}_{2} \mathrm{H}_{4}$ & 1,73 & 1,89 & & \\
\hline $\mathrm{CH}_{4}$ & 1,64 & 1,72 & & \\
\hline $\mathrm{N}_{2}$ & 1,54 & 1,62 & & \\
\hline $\mathrm{O}_{2}$ & 1,48 & 1,62 & & \\
\hline $\mathrm{F}_{2}$ & 1,39 & & & \\
\hline $\mathrm{H}_{2} \mathrm{O}$ & 1,20 & 1,34 & & \\
\hline $\mathrm{CF}_{4}$ & 1,66 & & & \\
\hline $\mathrm{p}-\mathrm{H}_{2}$ & 1,63 & $\begin{array}{l}1,87 \\
\end{array}$ & & \\
\hline${ }^{3} \mathrm{He}$ & 2,31 & 2,67 & & \\
\hline $\mathrm{C}_{3} \mathrm{H}_{8}$ & 1,89 & 2,04 & & \\
\hline
\end{tabular}

équations $(8 \mathrm{~b}),(8 \mathrm{~d}),(11 \mathrm{a}),(15 \mathrm{a})$ et $(16 \mathrm{a})$ à celles tirées d'autres déterminations expérimentales et phénoménologiques [35].

Dans le tableau III nous comparons, pour le $\mathrm{Xe}, \mathrm{SF}_{6}$ et $\mathrm{CO}_{2}$, les résultats de notre détermination de $\xi^{+}$, $D^{c}, B$ et $C^{+}$aux valeurs expérimentales correspondantes qui ont été à la base de la discussion précédente.

Les résultats de ces tableaux montrent que nos hypothèses définissant les deux paramètres critiques $Z_{\mathrm{c}}$ et $Y_{\mathrm{c}}$ comme nombres caractéristiques des comportements singuliers des fluides purs sont réalistes.

\section{Comportement singulier unique des potentiels thermodynamiques.}

Nous venons de supposer la séparation des rôles de chaque facteur caractéristique le long de deux lignes différentes de la surface $f(P, \rho, T)=0$. Par conséquent, la forme asymptotique d'au moins une propriété thermodynamique non-analytique définie sur une de ces lignes est entièrement caractérisée par le facteur qui lui est associé. En toute généralité, cette grandeur dérive de la contribution singulière d'un potentiel thermodynamique approprié. C'est pourquoi nous reformulons maintenant nos deux dernières hypothèses en considérant les comportements critiques respectifs de deux potentiels dont les choix sont justifiés par les analogies déjà soulignées entre les variables relatives au fluide pur et celles du système de spins [21].

Hypothèse 1 : Nous supposons que le comportement asymptotique le long de l'isochore critique du domaine monophasique, de l'écart, $\Delta P^{*}$ (réduit sans dimension) $d u$ potentiel thermodynamique $(P / T)$ par rapport à sa valeur au point critique, est le même pour tous les fluides purs, au seul facteur d'échelle $Y_{\mathrm{c}}$ près.

Hypothèse 2 : Nous supposons que le comportement asymptotique le long de l'isotherme critique de l'écart, $\Delta \mu^{*}$ (réduit sans dimension) du potentiel chimique $(\mu)$ par rapport à sa valeur au point critique, est le même pour tous les fluides purs, au seul facteur d'échelle $Z_{\mathrm{c}}$ près.

En vue de représenter le résultat prédit par les deux hypothèses 1 et 2 , nous considérons deux fluides purs différents I et II étiquetés $i$. Pour le chemin d'approche thermodynamique $(\mathrm{L})\{(\mathrm{L})=$ (isochore critique, isotherme critique) $\}$ le potentiel thermodynamique $G\{G=P / T, \mu\}$ est une fonction du champ $g\{g=T, \rho\}$. La figure 1a donne une allure schématique des courbes $\left.G_{i}(g)\right|_{(\mathrm{L})}$. Au point critique $\left(g=g_{\mathrm{c}}\right)$ le potentiel prend la valeur $G_{\mathrm{c}}$. Seuls sont intéressants les écarts de potentiel $\Delta G=G-G_{\mathrm{c}}$, fonctions de la distance en champ $\Delta g=g-g_{\mathrm{c}}$ mesurée le long de $(\mathrm{L})$.

$\mathrm{La}$ réduction sans dimension des grandeurs $\Delta G$ et $\Delta g$ fait intervenir les facteurs d'échelle $\beta_{\mathrm{c}}$ des énergies et a des longueurs (Eqs. (8a) et (8b)). Le résultat est schématisé sur la figure $1 \mathrm{~b}$ où les différentes courbes sont représentées par les fonctionnelles suivantes :

$$
\Delta G^{*}=\left.f_{i}^{*}\left(\Delta g^{*}\right)\right|_{(\mathrm{L})} .
$$


Tableau III. - Résultats relatifs au Xe, à $\mathrm{SF}_{6}$ et à $\mathrm{CO}_{2}$.

Lignes $(0)$ : coordonnées critiques généralisées.

Lignes (1) : paramètres caractéristiques définis par les équations (8).

Lignes (2) : Valeurs des amplitudes asymptotiques $\xi^{+}$et $D^{\mathbf{c}}$, calculées, à partir des paramètres caractéristiques, suivant la méthode proposée dans ce travail (Eqs. (11),(15) et (16)).

Lignes (3) : Valeurs calculées des amplitudes asymptotiques $\xi_{0}^{+}, D_{\mathrm{LSG}}^{\mathrm{c}}, B_{\mathrm{LSG}}$ et $C_{\mathrm{LSG}}^{+} ; \xi_{0}^{+}=a . \xi^{+}$. a est défini par l'équation (8b); $D_{\mathrm{LSG}}^{\mathrm{c}}, B_{\mathrm{LSG}}$ et $C_{\mathrm{LSG}}^{+}$sont définis dans la référence [20]. Voir aussi les références [30] et [34]. Pour déterminer $B_{\mathrm{LSG}}$ et $C_{\mathrm{LSG}}^{+}$il est fait usage des rapports universels suivants $: \boldsymbol{R}_{\chi} ; \boldsymbol{R}_{\xi}^{+} ; \boldsymbol{R}_{\mathrm{C}}$ et $R=R_{\xi}^{+} \cdot\left(R_{\mathrm{C}}\right)^{-1 / d}$ (voir Tableau I).

Les valeurs numériques retenues sont $R_{\chi}=1,7 ; R_{\xi}^{+}=0,27 ; R_{\mathrm{C}}=0,066 ; R=0,66811$ (voir le texte).

Lignes (4) : Valeurs expérimentales de $\xi_{0}^{+}$reprises du tableau II.

Lignes (5) : Valeurs de $D_{\mathrm{LSG}}^{\mathrm{c}}, B_{\mathrm{LSG}}$ et $C_{\mathrm{LSG}}^{+}$tirées de différentes analyses des résultats expérimentaux originaux de diffraction de Fraunhofer de la référence [25].

[Results for $\mathrm{Xe}, \mathrm{SF}_{6}$ and $\mathrm{CO}_{2}$.

Lines (0) : Generalized critical parameters of the pure fluid.

Lines (1): Caracteristic parameters of the pure fluid defined by equations (8).

Lines (2) : Calculated values of the asymptotic amplitudes, $\xi^{+}$and $D^{\mathrm{c}}$ from the caracteristic parameters using equations (11), (15) and (16).

Lines (3) $:$ Calculated values of the asymptotic amplitudes, $\xi_{0}^{+}, D_{\mathrm{LSG}}^{\mathrm{c}}, B_{\mathrm{LSG}}$ and $C_{\mathrm{LSG}}^{+} ; \xi_{0}^{+}=a . \xi^{+} . a$ is defined by equation (8b); $D_{\mathrm{LSG}}^{\mathrm{c}}, B_{\mathrm{LSG}}$ and $C_{\mathrm{LSG}}^{+}$are defined in reference [20]. See also the reference [30] and [34]. The calculations of $B_{\mathrm{LSG}}$ and $C_{\mathrm{LSG}}^{+}$take into account the following universal ratios $: R_{\chi} ; R_{\xi}^{+}$; $R_{\mathrm{C}}$ et $R=R_{\xi}^{+} .\left(R_{\mathrm{C}}\right)^{-1 / d}$ (see Table I). The numerical valuse used are $: R_{\xi}=1.7 ; R_{\xi}^{+}=0.27$; $R_{\mathrm{C}}=0.066 ; R=0.66811$ (see text).

Lines (4) : experimental values of $\xi_{0}^{+}$following table II.

Lines (5) : values of $D_{\mathrm{LSG}}^{\mathrm{c}}, B_{\mathrm{LSG}}$ obtained by different analysis of the data from the original Fraunhofer diffraction experiments in $\mathrm{Xe}, \mathrm{SF}_{6}$ and $\mathrm{CO}_{2}$ (Ref. [25]).]

\begin{tabular}{|c|c|c|c|c|c|}
\hline & $m$ & $\left(10^{-26} \mathrm{~kg}\right)$ & $\begin{array}{c}\mathrm{Xe} \\
21,803\end{array}$ & $\begin{array}{c}\mathrm{SF}_{6} \\
24,252\end{array}$ & $\begin{array}{l}\mathrm{CO}_{2} \\
7,308\end{array}$ \\
\hline (0) & $\begin{array}{l}P_{\mathrm{c}} \\
\rho_{\mathrm{c}} \\
T_{\mathrm{c}} \\
\gamma_{\mathrm{c}}^{\prime}\end{array}$ & $\begin{array}{l}(\mathrm{MPa}) \\
\left(\mathrm{kg} \cdot \mathrm{m}^{-3}\right) \\
(\mathrm{K}) \\
\left(\mathrm{MPa} \cdot \mathrm{K}^{-1}\right)\end{array}$ & $\begin{array}{c}5,84 \\
1110 \\
289,74 \\
0,119\end{array}$ & $\begin{array}{l}3,76 \\
737 \\
318,70 \\
0,0835\end{array}$ & $\begin{array}{c}7,3753 \\
467,8 \\
304,14 \\
0,170\end{array}$ \\
\hline & $\begin{array}{l}\beta_{\mathrm{c}}=k_{\mathrm{B}} T_{\mathrm{c}} \\
a=\left(k_{\mathrm{B}} T_{\mathrm{c}} / P_{\mathrm{c}}\right)^{1 / 3} \\
Z_{\mathrm{c}}=P_{\mathrm{c}} m / \rho_{\mathrm{c}} k_{\mathrm{B}} T_{\mathrm{c}} \\
Y_{\mathrm{c}}=\left(\gamma_{\mathrm{c}}^{\prime} T_{\mathrm{c}} / P_{\mathrm{c}}\right)-1\end{array}$ & $\begin{array}{l}\left(10^{-21} \mathrm{~J}\right) \\
\left(10^{-10} \mathrm{~m}\right)\end{array}$ & $\begin{array}{l}4,0 \\
8,815 \\
0,287 \\
4,9\end{array}$ & $\begin{array}{c}4,4 \\
10,54 \\
0,281 \\
6,08\end{array}$ & $\begin{array}{l}4,2 \\
8,29 \\
0,274 \\
6,01\end{array}$ \\
\hline & $\begin{array}{l}\xi^{+}=\left(Y_{\mathrm{G}} / Y_{\mathrm{c}}\right)^{v} \\
D^{\mathrm{c}}=\left(F_{\mathrm{G}} / Z_{\mathrm{c}}\right)^{-3(\delta+1) / 2}\end{array}$ & & $\begin{array}{c}0,1997 \\
5,04 \times 10^{-3}\end{array}$ & $\begin{array}{c}0,1745 \\
4,24 \times 10^{-3}\end{array}$ & $\begin{array}{c}0,175 \\
3,42 \times 10^{-3}\end{array}$ \\
\hline $\begin{array}{l}\text { (3) } \\
\text { (4) }\end{array}$ & $\begin{array}{l}\xi_{0}^{+}=a \cdot \xi^{+} \\
\xi_{0}^{+}(\exp )\left(\text { Tableau II) }\left\{\begin{array}{l}\text { Réf. [33] } \\
\text { Réf. [29] }\end{array}\right.\right.\end{array}$ & $\left(10^{-10} \mathrm{~m}\right)$ & $\begin{array}{c}1,76 \\
\left\{\begin{array}{l}1,86 \pm 0,10 \\
1,85 \pm 0,10\end{array}\right.\end{array}$ & $\begin{array}{c}1,84 \\
\left\{\begin{array}{l}1,86 \pm 0,10 \\
1,9 \pm 0,1\end{array}\right.\end{array}$ & $\begin{array}{c}1,45 \\
\left\{\begin{array}{l}1,50 \pm 0,10 \\
1,5 \pm 0,1\end{array}\right.\end{array}$ \\
\hline & $\begin{array}{l}D_{\mathrm{LSG}}^{\mathrm{c}}=Z_{\mathrm{c}}^{-(\delta+1)} \cdot D^{\mathrm{c}} \\
D_{\mathrm{LSG}}^{\mathrm{c}}\left\{\begin{array}{l}\operatorname{mini} \\
\operatorname{maxi}\end{array} \text { Analyse de la Réf. [19] }\right.\end{array}$ & & $\begin{array}{l}7,2 \\
6,13 \\
7,53\end{array}$ & $\begin{array}{l}6,8 \\
5,51 \\
7,10\end{array}$ & $\begin{array}{l}6,3 \\
5,92 \\
6,93\end{array}$ \\
\hline & $\begin{array}{l}B_{\mathrm{LSG}}=Z_{\mathrm{c}} \cdot\left[\left(R_{\chi} / D^{\mathrm{c}}\right) \cdot\left(R / \xi^{+}\right)^{d}\right]^{1 /(\delta+1)} \\
B_{\mathrm{LSG}}(\exp )\left\{\begin{array}{l}\text { Réf. [25] } \\
\text { Analyse de la Réf. [32] }\end{array}\right.\end{array}$ & & $\begin{array}{c}1,455 \\
1,48 \\
1,42 \pm 0,06\end{array}$ & $\left\{\begin{array}{c}1,575 \\
1,56 \\
1,62 \pm 0,06\end{array}\right.$ & $\left\{\begin{array}{c}1,59 \\
1,54 \\
1,59 \pm 0,05\end{array}\right.$ \\
\hline & $\begin{array}{l}C_{\mathrm{LSG}}^{+}=Z_{\mathrm{c}}^{2} \cdot\left(R_{\chi} / D^{\mathrm{c}}\right)^{2 /(\delta+1)} \cdot\left(\xi^{+} / R\right)^{2-\eta} \\
C_{\mathrm{LSG}}^{+}(\exp )\{\text { Analyse de la Réf. [32] }\end{array}$ & & $\begin{array}{c}0,0565 \\
\{0,058 \pm 0,002\end{array}$ & $\begin{array}{c}0,044 \\
\{0,046 \pm 0,004\end{array}$ & $\begin{array}{c}0,046 \\
\{0,046 \pm 0,002\end{array}$ \\
\hline
\end{tabular}




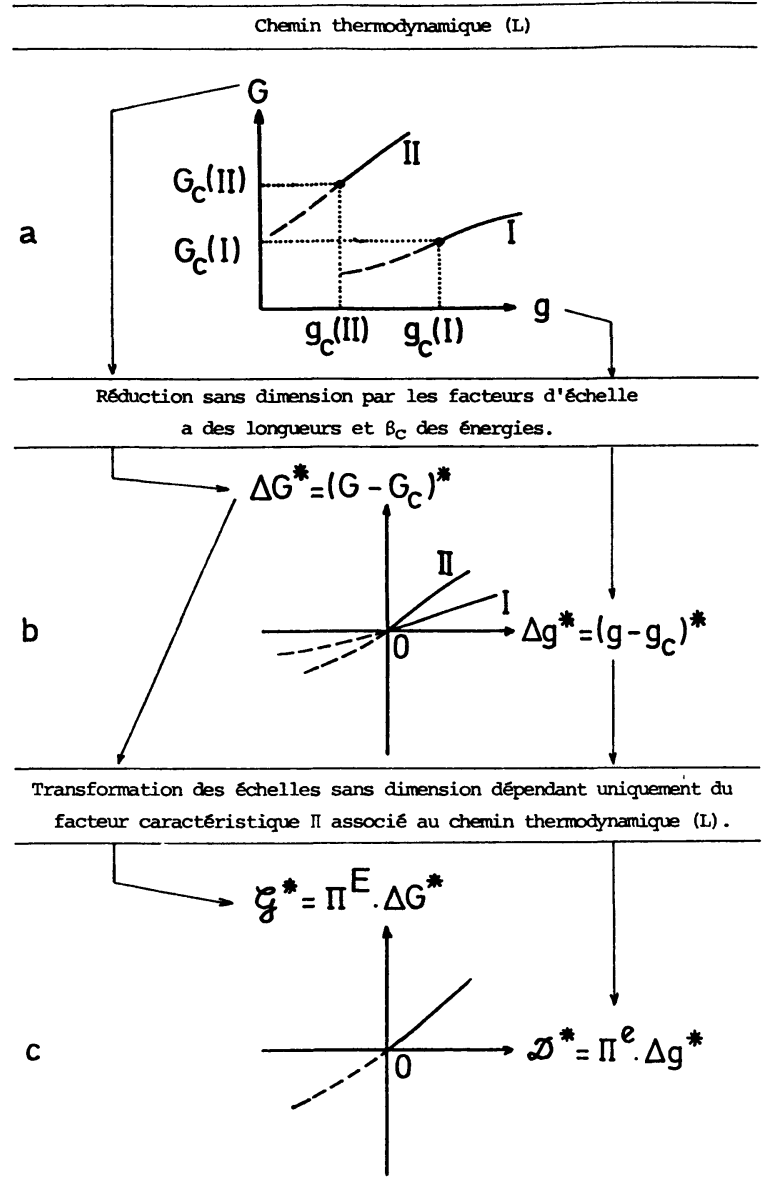

Fig. 1a. - Comportements asymptotiques le long de la ligne critique (L), du potentiel $G$ en fonction du champ $g$, pour les fluides purs I et II.

b. - Comportements asymptotiques réduits sans dimension des écarts du potentiel par rapport à sa valeur au point critique, en fonction de la distance en champ, pour les fluides purs I et II. La méthode de réduction fait intervenir séparément au niveau du champ $g$ et du potentiel $G$, soit le facteur d'échelle $a$ des longueurs (Eq. (8b)) soit celui $\beta_{\mathrm{c}}$ des énergies (Eq. (8a)).

c. - Comportement asymptotique unique, le long de (L), du potentiel renormalisé, pour l'ensemble de tous les fluides purs. Ce comportement est obtenu après une transformation des échelles des axes de la figure $1 \mathrm{~b}$ faisant appel au facteur d'échelle $\Pi$ associé à $(\mathrm{L}) ; \Pi$ est caractéristique de chaque fluide pur.

[a. - Asymptotic behaviours along the critical path $(\mathrm{L})$ of the thermodynamic potential $G$ as a function of the field $g$, for pure fluids I and II.

b. - Asymptotic behaviours of the dimensionless critical potential difference versus the dimensionless field distance to the critical point. The method of reduction to dimensionless quantities for the field $g$ and the potential $G$, involves separately either the length scaling factor $a$ (Eq. (8b)), or the energy one $\beta_{\mathrm{c}}$ (Eq. (8a)).

c. - Single asymptotic behaviour along $(\mathrm{L})$ of the renormalized potential for every pure fluids. This behaviour is obtained using the scale change of axis in figure $1 \mathrm{~b}$ which takes into account the scaling factor $\Pi$ related to $(\mathrm{L}) . \Pi$ is characteristic of each pure fluid.]
Suivant les hypothèses 1 et 2 , nous pouvons maintenant passer des courbes précédentes à une courbe unique dont l'équation s'écrit :

$$
\mathcal{S}_{\Pi}^{*}\left(\Delta G^{*}\right)=\mathcal{F}_{(\mathrm{L})}^{*}\left[\mathfrak{D}_{\Pi}^{*}\left(\Delta g^{*}\right)\right]
$$

où $\mathcal{F}_{(\mathrm{L})}^{*}$ est une fonction qui ne dépend plus du fluide pur considéré, mais seulement du chemin (L) suivi pour atteindre le point critique. Par contre, les nouvelles grandeurs réduites $\mathcal{S}_{I}^{*}$ et $\mathfrak{D}_{I}^{*}$ de l'équation (18) vont dépendre des grandeurs réduites expérimentales $\Delta G^{*}$ et $\Delta g^{*}$ au travers du nombre sans dimension $\Pi$ caractéristique de $(\mathrm{L})\left(\Pi=\left\{Y_{\mathrm{c}}\right.\right.$ pour $(\mathrm{L})=$ isochore critique ; $Z_{\mathrm{c}}$ pour $(\mathrm{L})=$ isotherme critique $\}$ ). Pour expliciter cette dépendance, nous adoptons le point de vue très général de la notion de dilatation d'échelle [36], caractérisée ici par $\Pi$. Les grandeurs $\Delta G^{*}$ et $\Delta g^{*}$ sont transformées dans la dilatation suivant les équations :

$$
\begin{aligned}
& \mathfrak{S}^{*}=\left.\Pi^{E} \cdot \Delta G^{*}\right|_{(\mathrm{L})} \\
& \mathfrak{D}^{*}=\left.\Pi^{e} \cdot \Delta g^{*}\right|_{(\mathrm{L})}
\end{aligned}
$$

où $E_{(\mathrm{L})}$ et $e_{(\mathrm{L})}$ ont les mêmes valeurs pour tous les fluides purs. Dans ces conditions, la figure 1c est représentative de la courbe unique le long de (L), décrite par l'équation (18), où les variables renormalisées $\mathcal{G}^{*}$ et $\mathfrak{D}^{*}$ sont obtenues à partir des variables physiques par les équations (19) et (20).

Pour mieux préciser la transformation d'échelle ainsi définie sur chaque chemin, nous redérivons maintenant des équations (17) à (20), les relations (11a) et (11b) du paragraphe précédent. Seules les contributions non-analytiques asymptotiques des différentes fonctions introduites ci-dessus sont nécessaires.

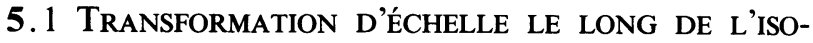
THERME CRITIQUE. - La forme d'échelle unique, $\mathfrak{F}^{*}$, de l'isotherme critique du potentiel chimique renormalisé, $\mathfrak{J C}^{*}$, est décrite par le terme suivant $[21,18]$ :

$$
\mathcal{H}^{*}=\Omega_{\mathrm{H}} \cdot\left|\mathcal{M}^{*}\right|^{\delta}
$$

où $\Lambda^{*}$ est le paramètre d'ordre renormalisé. $\Omega_{\mathrm{H}}$ est un nombre qui a la même valeur pour tous les fluides purs. En accord avec les équations (19) et (20), nous définissons maintenant les grandeurs renormalisées le long de l'isotherme critique par :

$$
\begin{aligned}
\mathscr{H}^{*} & =Z_{\mathrm{c}}^{V} \cdot \Delta \mu^{*} \\
\mathcal{M}^{*} & =Z_{\mathrm{c}}^{v} \cdot \Delta \rho^{*} .
\end{aligned}
$$

L'amplitude asymptotique $D^{\mathrm{c}}$, (voir l'Eq. (3)), qui correspond aux grandeurs physiques réduites sans dimension étant définie par :

$$
\Delta \mu^{*}=D^{\mathrm{c}}\left|\Delta \rho^{*}\right|^{\delta},
$$

nous obtenons la relation :

$$
D^{\mathrm{c}}=\Omega_{\mathrm{H}} \cdot Z_{\mathrm{c}}^{r \delta-V},
$$


qui se met sous la forme :

$$
\left(D^{\mathrm{c}}\right)^{1 /(V-\imath \delta)} \cdot Z_{\mathrm{c}}=\Omega_{\mathrm{H}}^{1 /(V-\imath \delta)}
$$

équivalente à celle de l'équation (11b).

Les valeurs de $V$ et $v$ sont liées par l'intermédiaire de $\delta$ lorsque nous tenons compte de l'équation (15b) dans l'identification terme à terme des équations (26) et (11b). Ce qui complète nos définitions, par les équations (22) et (23), des variables renormalisées intervenant dans l'équation (21); le long de l'isotherme critique la renormalisation d'une seule variable suffit à caractériser la dilatation d'échelle associée à ce chemin. Les deux nouveaux nombres introduits dans ce paragraphe ( $\Omega_{\mathrm{H}}$ d'une part et l'un des deux exposants $V$ ou $v$ d'autre part) sont respectivement reliés aux nombres $F_{\mathrm{G}}$ et $\zeta$ introduits précédemment par la relation (11b). Mais le fait que, dans le paragraphe 4, nous n'ayons pas été en mesure de définir avec certitude la valeur unique de l'exposant $\zeta$ (ou, de manière équivalente ici, celle de l'un des deux exposants $V$ ou $v$ ) empêche d'aller plus loin dans notre approche phénoménologique de cette dilation à $T=T_{\mathrm{c}}$.

5. 2 Transformation D'ÉCHELle LE LONG DE L'ISOCHORE CRITIQUE $\left(T>T_{\mathrm{c}}\right)$. - En approchant du (PCLV) le long de l'isochore critique dans le domaine monophasique, il est cette fois possible de préciser quantitativement la transformation d'échelle associée à ce chemin. Les écarts réduits sans dimension des grandeurs physiques concernées s'écrivent, en termes de la pression et de la température, de la manière suivante :

$$
\begin{aligned}
\Delta P^{*} & =\left(P T_{\mathrm{c}} / T P_{\mathrm{c}}\right)-1 \\
\Delta t^{*} & =\tau=\left(T / T_{\mathrm{c}}\right)-1 .
\end{aligned}
$$

En accord avec notre hypothèse 1 et les notations adoptées, les variables renormalisées sont définies par les relations qui suivent :

$$
\begin{aligned}
& \mathfrak{T}^{*}=Y_{\mathrm{c}}^{W} \cdot \Delta P^{*} \\
& \mathfrak{C}^{*}=Y_{\mathrm{c}}^{w} \cdot \Delta t^{*} .
\end{aligned}
$$

Pour la classe $(3,1)$, le terme non-analytique asymptotique de la forme universelle du potentiel thermodynamique défini le long de l'isochore critique est en $\left(\mathcal{C}^{*}\right)^{2-\alpha}[21,23]$. Pour la sous-classe particulière des fluides purs, l'expérience montre la prédominance dans $\Delta P^{*}$ d'un terme linéaire en $\Delta t *$ qui justifie d'ajouter une contribution régulière au potentiel renormalisé. En ne considérant que les termes dominants régulier et non-analytique, la forme d'échelle unique du potentiel $\mathfrak{S}^{*}$ doit s'écrire :

$$
\mathfrak{T}^{*}=a_{1} \cdot \mathfrak{C}^{*}+A_{0} \cdot\left(\mathfrak{C}^{*}\right)^{2-\alpha},
$$

$a_{1}$ et $A_{0}$ ayant même valeur respective pour tous les fluides purs. Compte-tenu de la signification du paramètre caractéristique $Y_{\mathrm{c}}$, (voir l'Eq. (8d)), le choix des valeurs $W=0$ et $w=1$ dans les équations (29) et (30) implique la valeur $a_{1}=1$ quel que soit le fluide pur considéré. Nous prenons donc comme nouvelle définition des variables renormalisées :

$$
\begin{aligned}
& \mathfrak{T}^{*}=\Delta P^{*} \\
& \mathcal{C}^{*}=Y_{\mathrm{c}} \cdot \Delta t^{*}=Y_{\mathrm{c}} \cdot \tau
\end{aligned}
$$

où $Y_{\mathrm{c}}$ joue le rôle de facteur multiplicatif pour la distance réduite habituelle aux expérimentateurs. De son côté, le potentiel thermodynamique renormalisé à $\rho=\rho_{\mathrm{c}}$ s'identifie exactement à l'écart réduit du potentiel réel.

Avec ce choix particulier, l'obtention de la relation (11a) admise au paragraphe précédent est maintenant exacte. En effet, le comportement singulier de la chaleur spécifique réduite $C^{*}$ est proportionnel au comportement singulier de la dérivée seconde du potentiel considéré ci-dessus. L'amplitude asymptotique $A^{+}$, correspondant aux variables physiques, est définie par la loi de divergence suivante :

$$
C^{*}=\frac{A^{+}}{\alpha} \cdot(\tau)^{-\alpha} .
$$

Par conséquent, l'identification de l'équation (34) à la dérivée seconde par rapport à la température réduite de l'équation (31), où il est tenu compte des équations (32) et (33), conduit à une relation entre $A^{+}$et $Y_{\mathrm{c}}$ ne faisant intervenir que des nombres universels de la classe $(3,1)$ ou ayant même valeur pour la sous-classe des fluides purs. Cette relation prend la forme qui suit :

$$
A^{+}=\Omega_{\mathrm{C}} \cdot Y_{\mathrm{c}}^{2-\alpha}
$$

où $\Omega_{\mathrm{C}}$ a une valeur unique pour tous les fluides purs ( $\Omega_{\mathrm{C}}$ ne dépend que des valeurs unique de $A_{0}$ et universelle de $\alpha$ ).

Sachant que le GR prévoit l'existence du rapport universel $R_{\xi}^{+}$(Tableau I), de l'équation (35) nous déduisons le résultat suivant :

$$
\left(\xi^{+}\right)^{d /(2-\alpha)} \cdot Y_{\mathrm{c}}=\left(R_{\xi}^{+}\right)^{d /(2-\alpha)} \cdot \Omega_{\mathrm{C}}^{1 /(\alpha-2)} .
$$

L'équation (36) est équivalente à l'équation (11a) du fait de la loi d'échelle de Josephson entre les exposants critiques $\alpha$ et $v$ (Tableau I).

La formulation et la démarche suivies dans ce paragraphe pour retrouver les équations (11) caractérisent des transformations non-universelles des échelles physiques. Les transformations effectuées, nous prévoyons, asymptotiquement au (PCLV), un comportement d'échelle unique d'une propriété singulière des fluides purs sur chaque chemin thermodynamique considéré. Ces résultats phénoménologiques sont donc comparables à ceux prédits par Bagnuls et Bervillier [10].

\section{Comparaison avec les résultats théoriques de Bagnuls et Bervillier [10].}

Le long de l'isochore critique des fluides purs, nous proposons une transformation d'échelle dont la défi- 
nition phénoménologique,

$$
\mathcal{C}^{*}=Y_{\mathrm{c}} \cdot \tau(\text { rappel de l'Eq. (33)), }
$$

est formellement équivalente à celle de l'approche fondamentale du (GR),

$$
t^{*}=\theta . \tau \quad \text { (rappel de l'Eq. (4)) }
$$

Suivant l'application de Bagnuls et Bervillier, le comportement singulier de la longueur de corrélation réduite sans dimension, dépend uniquement de la variable d'échelle $t^{*}$. Le terme asymptotique de ce comportement s'écrit :

$$
l^{*}\left(t^{*}\right)=3_{\xi}^{*} \cdot\left(t^{*}\right)^{-v} \cdot\left[1+0\left(t^{*}\right)\right]
$$

où $3_{\xi}^{*}$ est une constante. L'identification des équations (2) et (37) en tenant compte de l'équation (4), conduit à la relation suivante :

$$
\xi^{+}=3_{\xi}^{*} \cdot \theta^{-v}
$$

L'équation (38) est comparable à notre équation phénoménologique (11a) mise sous la forme qui suit :

$$
\xi^{+}=\tilde{\zeta}_{\xi} \cdot\left(Y_{c}\right)^{-1 / \phi}
$$

d'où la relation évidente $\phi=1 / v$ entre les exposants (Eq. (15a)). Par contre, la valeur de la constante phénoménologique,

$$
\tilde{3}_{\xi}=\left(Y_{\mathrm{G}}\right)^{v}
$$

égale à $\tilde{\zeta}_{\xi}=0,54$ d'après l'équation (16a), diffère de celle calculée, $3_{\varepsilon}^{*}=0,48$, dans le traitement numérique $\operatorname{de}\left(\phi^{4} 3 \mathrm{D}\right)$. Cette différence est due essentiellement à la non-universalité des systèmes de réduction sans dimension des grandeurs physiques.

Dans l'équation (37) obtenue à partir du modèle théorique, la non-universalité qui caractérise un système $(S)$ donné, n'apparaît pas de façon explicite. Cependant il est aisé de concevoir que la réduction des variables physiques de (S) prend en compte une partie de cette non-universalité, tandis que l'autre partie viendra de l'hypothèse de base du (GR). Ce point a été souligné par Bagnuls et Bervillier [10], et discuté, pour le cas précis où le système $(\mathrm{S})$ était le xénon [17].

Dans l'équation (39), nous avons séparé la nonuniversalité d'un fluide pur en deux termes : le nombre sans dimension $\widetilde{\zeta}_{\xi}$ est associé aux deux unités choisies pour exprimer en variables réduites, les grandeurs physiques relatives à ce fluide pur; $Y_{\mathrm{c}}$ est le facteur d'échelle non-universel caractéristique de l'isochore critique. A la condition de conserver la même définition des unités dimensionnées quel que soit le fluide pur étudié, notre approche suppose alors que $\tilde{\widetilde{\zeta}}_{\xi}$ garde la même valeur numérique pour tous les fluides purs. Mais sans élément de comparaison entre les systèmes de réduction propres au modèle $\left(\phi^{4} 3 \mathrm{D}\right)$ et aux fluides, il n'y a pas de raison évidente pour que la valeur de $\widetilde{\zeta}_{\xi}$ soit universelle et donc égale à la valeur $\zeta_{\xi}^{*} \operatorname{de}\left(\phi^{4} 3 \mathrm{D}\right)$.

\section{Conclusion.}

Nous avons supposé que le comportement singulier asymptotique d'un fluide pur à l'équilibre au voisinage de son point critique liquide-vapeur est entièrement caractérisé par quatre paramètres macroscopiques. Ces paramètres sont dérivés de la localisation de son point critique sur la surface caractéristique $f(P, \rho, T)=0$ et du plan tangent en ce point à cette surface. Deux de ces paramètres, $\beta_{\mathrm{c}}=k_{\mathrm{B}} T_{\mathrm{c}}$ et $a=\left(k_{\mathrm{B}} T_{\mathrm{c}} / P_{\mathrm{c}}\right)^{1 / 3}$, sont les facteurs des échelles dimensionnées habituelles, définissant respectivement une énergie et une longueur caractéristiques de ce point. Les deux autres, $Z_{\mathrm{c}}=P_{\mathrm{c}} m / \rho_{\mathrm{c}} k_{\mathrm{B}} T_{\mathrm{c}}$ et $Y_{\mathrm{c}}=\left(\gamma_{\mathrm{c}}^{\prime} T_{\mathrm{c}} / P_{\mathrm{c}}\right)-1$, sont deux nombres sans dimension.

Afin de rendre valide l'universalité à deux facteurs d'échelle près prédite par la description théorique du GR, nous avons proposé deux rapports qui relient respectivement les amplitudes critiques asymptotiques $\xi^{+}$et $D^{\mathrm{c}}$ aux deux nombres précédents $Y_{\mathrm{c}}$ et $Z_{\mathrm{c}}$. Nous fermons ainsi correctement le nouvel ensemble constitué par les 12 amplitudes asymptotiques théoriques de la classe d'universalité $d=3, n=1$ et les deux nombres expérimentaux précédents $Y_{\mathrm{c}}$ et $Z_{\mathrm{c}}$. En conséquence, le comportement singulier de chaque fluide pur critique - qui est entièrement défini au sens du GR par les deux amplitudes $\xi^{+}$et $D^{\mathfrak{c}}$ - est bien caractérisé par les quatre paramètres $\beta_{\mathrm{c}}, a, Y_{\mathrm{c}}$ et $Z_{\mathrm{c}}$ (ou les coordonnées $P_{\mathrm{c}}, \rho_{\mathrm{c}}, T_{\mathrm{c}}$ et $\gamma_{\mathrm{c}}^{\prime}$ ).

Nous avançons ensuite deux nouvelles hypothèses d'unicité de comportement des potentiels thermodynamiques singuliers définis respectivement le long de l'isochore critique et le long de l'isotherme critique. A partir de ces hypothèses nous proposons une méthode de transformation d'échelle des champs physiques réduits mesurant la distance au point de transition sur chacun de ces chemins. Nous obtenons alors simplement les relations entre les deux amplitudes asymptotiques $\xi^{+}$et $D^{\text {c }}$ laissées libres par le GR et les nombres caractéristiques expérimentaux $Y_{\mathrm{c}}$ et $Z_{\mathrm{c}}$, sous leur forme retenue précédemment. Une comparaison avec les résultats d'une application non-linéaire de la théorie du GR montre que la méthode proposée de transformation des échelles des champs physiques est équivalente à celle utilisée dans cette approche théorique.

Nous sommes maintenant en mesure de tester l'universalité associée aux autres contributions théoriques, comme celle récemment prévue à propos du premier terme non-analytique de correction au comportement asymptotique [10].

\section{Remerciements.}

Nous remercions R. Tufeu et B. Le Neindre de leur aide et de leurs encouragements au cours de ce travail. Nous remercions également C. Bervillier et C. Bagnuls pour les commentaires et les explications enrichissants à propos de leurs développements récents d'une application du GR sur le modèle $\phi^{4}$ à trois dimensions. 


\section{Bibliographie}

[1] Phase Transitions, status of the experimental and theoretical situation, Cargèse 1980, Eds. M. Levy, J. C. Le Guillou and J. Zinn-Justin (Plenum Press, N.Y.) 1982.

[2] Kadanoff, L. P., Critical Phenomena, Enrico Fermi Course LI, Ed. M. S. Green (Academic Press, N.Y.) 1971, p. 100 ;

Griffiths, R. B., Phys. Rev. Lett. 24 (1970) 1479.

[3] Baker Jr, G. A., Nickel, B. G., Green, M. S. and MeIron, D. I., Phys. Rev. Lett. 36 (1976) 1351;

BAKER Jr, G. A., NiCKel, B. G. and Meiron, D. I., Phys. Rev. B 17 (1978) 1365.

[4] Le Guillou, J. C. and Zinn-Justin, J., Phys. Rev. Lett. 39 (1977) 95; Phys. Rev. B 21 (1980) 3976.

Zinn-Justin, J., J. Physique 40 (1979) $969 ; 42$ (1981) 783.

[5] Bervillier, C. and Godrèche, C., Phys. Rev. B 21 (1980) 5427.

Aharony, A. and Hohenberg, P. C., Phys. Rev. B 13 (1976) 3081.

[6] Bagnuls, C. and Bervillier, C., Phys. Rev. B 24 (1981) 1226.

[7] Stauffer, D., Ferer, M. and Wortis, M., Phys. Rev. Lett. 29 (1972) 345 ;

Hohenberg, P. C., Aharony, A., Halperin, B. I. and Siggia, E. D., Phys. Rev. B 13 (1976) 2986 ;

Bervillier, C., Phys. Rev. B 14 (1976) 4964.

[8] Lee, T. D. and Yang, C. N., Phys. Rev. 87 (1952) 410.

[9] Fisher, M. E., Rep. Progr. Phys. 30 (1967) 615;

Sengers, J. V. and Levelt-Sengers, J. M. H., Progress in Liquid Physics, Ed. C. A. Croxton (Wiley, N.Y.) 1978, p. 103.

[10] Bagnuls, C. and Bervillier, C., J. Physique Lett. 45 (1984) L-95.

[11] Wegner, F. J., Phys. Rev. B 5 (1972) 4529.

[12] Le fait de ne pas prendre en compte les prévisions relatives aux corrections non-analytiques n'affecte en rien la démarche qui est suivie dans le présent travail.

[13] Bervillier, C., dans la Réf. [7].

[14] Voir par exemple : Levelt-Sengers, J. M. H. and Sengers, J. V., Perspectives in Statistical Physics, Ed. H. J. Raveché (North Holland Publ. Comp., Amsterdam) 1981, p. 239.

[15] Les notations et les définitions de toutes les grandeurs qui n'ont pas été explicitées dans notre travail sont celles de la Réf. [13].

[16] Wilson, K. G., Phys. Rev. B 4 (1971) 3174, 3184 ; Wilson, K. G. and Kogut, J., Phys. Rep. 12C (1974) 77.

[17] Bagnuls, C., Bervillier, C. and Garrabos, Y., J. Physique Lett. 45 (1984) L-127.

[18] Sengers, J. V., dans la Réf. [1], p. 95.

[19] Garrabos, Y., Thèse, Paris (1982).

[20] Ce n'est pas la seule façon possible pour réduire sans dimension les propriétés physiques d'un fluide pur. A titre d'exemple, nous donnons ici, pour toutes les amplitudes critiques asymptotiques utilisées dans le présent travail, les relations entre celles exprimées en unités de $\beta_{\mathrm{c}}$ (Eq. (8a)), et de a (Eq. (8b)), et celles résultant du système de réduction sans dimension adopté par J. M. H. LeveltSengers, W. L. Greer et J. V. Sengers (J. Phys. Chem. Ref. Data 5 (1976) 1), Réf. [30]; ces dernières sont affectées de l'indice LSG.

$\xi^{+}=\xi_{\mathrm{LSG}}^{+} ; \quad A^{+}=A_{\mathrm{LSG}}^{+} ; \quad \hat{D}=\hat{D}_{\mathrm{LSG}} \cdot Z_{\mathrm{c}}^{-2} ;$

$D^{\mathrm{c}}=D_{\mathrm{LSG}}^{\mathrm{c}} \cdot Z_{\mathrm{c}}^{\delta+1} ; \quad B=B_{\mathrm{LSG}} \cdot Z_{\mathrm{c}}^{-1} ; \quad C^{+}=C_{\mathrm{LSG}}^{+} \cdot Z_{\mathrm{c}}^{-2}$.

[21] Ley-Koo, M. and Green, M. S., Phys. Rev. A 16 (1977) 2483 ; A 23 (1981) 2650.

[22] Voir par exemple : Fisher, M. E. dans la Réf. [9] ; Fisher, M. E. and Burford, R. J., Phys. Rev. 156 (1967) 583 ;

Domв, C., Phase Transitions and Critical Phenomena, Eds. C. Domb and M. S. Green (Academic Press, N.Y.) 1974, Vol. 3, p. 357 et les références citées.

[23] Moldover, M. R., dans la Réf. [1], p. 63.

[24] Greywall, D. S. and Ahlers, G., Phys. Rev. A 7 (1973) 2145 ;

Ahlers, G., Phys. Rev. A 8 (1973) 530 ; voir aussi la Réf. [14].

[25] Hocken, R. and Moldover, M. R., Phys. Rev. Lett. 37 (1976) 29.

[26] Lunacek, J. H. and Cannell, D. S., Phys. Rev. Lett. 27 (1971) 841.

[27] Cannell, D. S., Phys. Rev. A 12 (1975) 225.

[28] Güttinger, H. and Cannell, D. S., Phys. Rev. A 24 (1981) 3188.

[29] Garrabos, Y., Tufeu, R. et Le Neindre, B., résultats non publiés.

[30] Levelt-Sengers, J. M. H., Greer, W. L. and SeNGers, J. V., J. Phys. Chem. Ref. Data 5 (1976) 1.

[31] Les études expérimentales au voisinage du (PLCV) du xénon sont les plus complètes qui aient été effectuées à ce jour sur un fluide pur critique. La vérification de l'universalité à deux facteurs d'échelle près a pu se faire sur plusieurs rapports universels. A ce propos, voir les références [17], [19], [25], et les deux références qui suivent.

[32] Sengers, J. V. and Moldover, M. R., Phys. Lett. 66A (1978) 44.

[33] Sengers, J. V., dans la référence [1], p. 124.

[34] Les valeurs expérimentales que nous avons retenues sont respectivement : $B_{\mathrm{LSG}}(\mathrm{Xe})=1,455$ et $C_{\mathrm{LSG}}^{+}(\mathrm{Xe})=0,0565$. Les relations qui permettent d'obtenir les valeurs correspondantes de $B$ et $C^{+}$ en fonction de $B_{\mathrm{LSG}}$ et de $C_{\mathrm{LSG}}^{+}$sont données dans la référence [20].

[35] Sengers, J. V., communication privée ;

Sengers, J. V. and LeVelt-Sengers, J. M. H., référence [9], p. 144.

[36] Toulouse, G. et Pfeuty, P., Introduction au Groupe de Renormalisation (P.U. Grenoble) 1975. 